\title{
EUCLIDEAN $(q+r)$-SPACE MODULO AN $r$-PLANE OF COLLAPSIBLE $p$-COMPLEXES $\left({ }^{1}\right)$
}

\author{
BY \\ LESLIE C. GLASER $\left({ }^{2}\right)$
}

\begin{abstract}
The following general decomposition result is obtained: Suppose $K^{p}(p \geqq 1)$ is a finite collapsible $p$-complex topologically embedded as a subset of a separable metric space $X^{q}$ where, for some $r \geqq 1, X^{q} \times E^{r}$ is homeomorphic to Euclidean $(q+r)$-space $E^{q+r}$. Then the Cartesian product of the quotient space $X^{q} / K^{p}$ with $E^{r}$ is topologically $E^{q+r}$ provided that $q \geqq 3$ and, for each simplex $\Delta^{k} \in K^{p}$, $\left(X^{q} \times E^{r}, \Delta^{k} \times\left([0,1]^{r-1} \times 0\right)\right)$ is homeomorphic, as pairs, to

$$
\left(E^{q+r},[0,1]^{k+r-1} \times(0, \ldots, 0)\right) \text {. }
$$

It is known that this condition is satisfied if $q-p \geqq 2$ and $q+r \geqq 5$. This result implies that if $K^{k}$ is a finite collapsible $k$-complex topologically embedded as a subset of Euclidean $n$-space $E^{n}$, then the Cartesian product of the quotient space $E^{n} / K^{k}$ with $E^{1}$ is topologically $E^{n+1}$ provided either (i) $n \leqq 3$, (ii) $n-k \geqq 2$, or (iii) each simplex of $K^{k}$ is flat in $E^{n+1}$.
\end{abstract}

0 . Introduction and definitions. In [1], by using a result of Klee [14] and some of the techniques developed by Bing in [2], Andrews and Curtis proved that if $A$ is an arc in Euclidean $n$-space $E^{n}$, then the Cartesian product of the quotient space $E^{n} / A$ with $E^{1}$ is topologically $E^{n+1}$. The significant aspects of this result appear when $n \geqq 3$, for in this case $E^{n} / A$ is not necessarily homeomorphic to $E^{n}$.

In [3], Bryant proves the following result:

TheOREM 0.1. Suppose that $D^{k}$ is a $k$-cell topologically embedded in $E^{n}$. Then $\left(E^{n} / D^{k}\right) \times E^{1}$ is homeomorphic to $E^{n+1}$ provided

I. $D^{k}$ is flat in $E^{n+1}$, or

II. $n-k \geqq 2$.

In proving this result under Condition II, Bryant made use of Homma's Approximation Theorem ([12], which later was found to contain a gap) to homeomorphically approximate $D^{k}$ in $E^{n+1}$ by a piecewise-linear $k$-cell. However, by recent results obtained in [8] or [16], this approximation may now be obtained,

Received by the editors May 1, 1970 and, in revised form, August 17, 1970.

AMS 1969 subject classifications. Primary 5478, 5705; Secondary 5425, 5525.

Key words and phrases. Euclidean space, $p$-cell, collapsible complex, separable metric space, quotient space, Cartesian product, a map bounded on the $E^{r}$ factor, $k$-flat, $r$-fold suspension, pseudo-isotopy

(1) Work partially supported by the National Science Foundation under NSF grant GP-11478.

(2) Alfred P. Sloan Fellow.

Copyright (C) 1971, American Mathematical Society 
and Bryant's generalization of the techniques of [1] holds as stated. In fact, at the time of this writing, it has been shown that a $k$-cell $D$ in $E^{n}$ is always flat in $E^{n+1}$ except possibly when $n \geqq 4$ and $k=n-1$. (See [14], [11], [6] and [7].) Moreover, just recently in [4], Bryant has shown that if $D$ is an $(n-1)$-cell topologically embedded in $E^{n}(n \geqq 4)$, then $\left(E^{n} / D\right) \times E^{1} \approx E^{n+1}$. The proof given in [4] does not require a $\mathrm{PL}$ approximation theorem, but makes use of radial engulfing and can actually be used for any $k=1,2, \ldots, n$ so long as $n \geqq 4$.

In [10], in studying suspensions of PL homology $n$-spheres and simplicial triangulations of topological manifolds, the author proved the following generalization of the main result of [1]:

THEOREM 0.2. Suppose $A$ is an arc in a separable metric space $X^{q}$ and $A$ has end points $x_{1}$ and $x_{2}$ such that

(a) $X^{q}-\left(x_{1} \cup x_{2}\right)$ is an open $q$-manifold $(q \geqq 3)$,

(b) $A-\left(x_{1} \cup x_{2}\right)$ is locally flat in $X^{q}-\left(x_{1} \cup x_{2}\right)$, and

(c) $X^{q} \times E^{r}$ is topologically homeomorphic to $E^{q+r}(r \geqq 1)$.

Then $\left(X^{q} / A\right) \times E^{r} \approx X^{q} \times E^{r} \approx E^{q+r}$ ( $\approx$ denotes "topologically homeomorphic"). Moreover, if $r=1$ (and $X^{q} \times E^{1} \approx E^{q+1}$ ), then properties (a) and (b) may be replaced by the single assumption that $A$ is flat in $X^{q} \times E^{1}$.

This latter conclusion follows directly from the work done in [1]. The results here are motivated by (i) the author's feeling that neither properties (a) and (b), nor the assumption that $A$ is flat in $X^{q} \times E^{r}$ (even if $r \neq 1$ ), are really necessary in the hypothesis of Theorem 0.2 to obtain the same conclusion, and by (ii) the following conjecture, which gives one natural generalization of Theorem 0.1 :

CONJECTURE 0.3. Suppose that $K^{k}$ is a finite collapsible $k$-complex topologically embedded in $E^{n}$. If each simplex of $K^{k}$ is flat in $E^{n+1}$, then $\left(E^{n} / K^{k}\right) \times E^{1}$ is homeomorphic to $E^{n+1}$.

The work done here is based on the simple observation that Theorem 0.1 holds if we replace $E^{n}$ by a separable metric space $X^{n}$ such that $X^{n} \times E^{1} \approx E^{n+1}$, and uses quite heavily the ideas and methods of [3]. We also may replace condition I and II by the single condition that $D^{k}$ is flat in $X^{n} \times E^{1} \approx E^{n+1}$. That is, if $X^{n} \approx E^{n}$, then $D^{1}$ is flat in $E^{n} \times E^{1}$ [14] and $D^{k} \subset E^{3}(k=2$ or 3$)$ is flat in $E^{3} \times E^{1}$ [11]. If $X^{n} \times E^{1} \approx E^{n+1}(n \geqq 4)$, then $D^{k}$ is flat in $X^{n} \times E^{1}$ provided either $k \leqq n-2$ [6] or $k=n[7]$.

Here, we show that Conjecture 0.3 is true and that Theorem 0.2 is valid without assuming either properties (a) and (b), or that $A$ is flat in $X^{q} \times E^{r}$ (for $q=3$ and $r=1$, we still seem to require that $A$ is flat in $X^{3} \times E^{1} \approx E^{4}$ ). In fact, we completely extend each to the following general result:

THEOREM 0.4. Suppose $K^{p}$ is a finite collapsible p-complex topologically embedded as a subset of a separable metric space $X^{q}$, where $X^{q} \times E^{r} \approx E^{q+r}, q \geqq 3$, and $r \geqq 1$. If for each simplex $\Delta^{k} \in K^{p}(k=1,2, \ldots, p), \Delta^{k} \times[0,1]^{r-1} \times 0\left(\subset X^{q} \times E^{r-1} \times 0\right)$ is flat in $X^{q} \times E^{r}$, then $\left(X^{q} / K^{p}\right) \times E^{r} \approx X^{q} \times E^{r} \approx E^{q+r}$. 
Actually, when we prove this result, the homeomorphism we obtain between $\left(X^{q} / K^{p}\right) \times E^{r}$ and $X^{q} \times E^{r}$ is natural in that given $\varepsilon>0$, we can construct a homeomorphism that is the "identity" outside an $\varepsilon$-neighborhood of $K^{p} \times E^{r}$ and changes $E^{r}$ coordinates less than $\varepsilon$. (Refer to Theorem 1.6 for the precise statement of this result.)

Making use of [14], [11], and [6], we immediately obtain the following result:

COROLlaRY 0.5. If $K^{p}$ is a finite collapsible p-complex topologically embedded in $E^{n}$ and either $n=3$ and $p \leqq 3$, or $n \geqq 4$ and $p \leqq n-2$, then $\left(E^{n} / K^{p}\right) \times E^{1} \approx E^{n+1}$.

We note that Corollary 0.5 follows very quickly from the observation and discussion given in the paragraph following Conjecture 0.3. That is, simply use induction on the number of moves required to collapse $K^{p}$ to a point.

We also obtain some additional related results. These are given at the end of $\S 1$. We now give some definitions and notation.

Given integers $s$ and $t, 0 \leqq s \leqq t$ and $t \geqq 1$, let $I^{s} \subset E^{t}$ (Euclidean $t$-space) be defined by

$$
\left.I^{s}=\left\{x_{1}, \ldots, x_{t}\right) \mid 0 \leqq x_{i} \leqq 1 \text { for } i \leqq s, \text { and } x_{i}=0 \text { for } s<i \leqq t\right\} .
$$

Suppose $F^{p}$ is a $p$-cell $\left(F^{p} \approx[0,1]^{p} \approx I^{p}\right)$ embedded as a subset of a separable metric space $X^{q}$, where $X^{q} \times E^{r} \approx E^{q+r}$. If $r \geqq 1$ and $0 \leqq k \leqq r$, we say $F^{p}$ is $k$-flat in $X^{q} \times E^{r}$ if $\left(E^{q+r}, I^{p+k}\right) \approx\left(X^{q} \times E^{r}, F^{p} \times I^{k}\right)$. If $r \geqq 0$ and $X^{q} \times E^{r} \approx E^{q+r}$, we say that $F^{p}$ is flat in $X^{q} \times E^{r}$ if $F^{p}$ is 0-flat in $X^{q} \times E^{r}$ (where for $r=0, X^{q} \times E^{0}=X^{q}$ ). Thus $F^{p}$ is $k$-flat in $X^{q} \times E^{r} \approx E^{q+r}$, if $F^{p} \times I^{k}$ is flat in $X^{q} \times E^{r}$.

If $w=\left(w_{1}, \ldots, w_{r}\right)$ is a point of $E^{r}$, then $\|w\|=\left(w_{1}^{2}+w_{2}^{2}+\cdots+w_{r}^{2}\right)^{1 / 2}$. We will use the usual metric induced on $E^{r}$ by this norm \|\| . If $Y$ is an arbitrary metric space with metric $d$ and $A$ is a subset of $Y$, then given $\varepsilon>0$

$$
U_{\varepsilon}(A, Y)=\{y \in Y \mid d(y, A)<\varepsilon\} .
$$

If $A$ is a compact set in $Y$, by the diameter of $A$ we will mean the number

$$
\operatorname{diam} A=\sup \left\{d\left(a_{1}, a_{2}\right) \mid a_{1} \in A \text { and } a_{2} \in A\right\} .
$$

By the Cartesian product metric on $Y \times E^{r}$, we will mean the metric $D$ on $Y \times E^{r}$ defined as follows:

$$
D\left(\left(y_{1}, w_{1}\right),\left(y_{2}, w_{2}\right)\right)=\left\{\left[d\left(y_{1}, y_{2}\right)\right]^{2}+\left[\left\|w_{1}-w_{2}\right\|\right]^{2}\right\}^{1 / 2} .
$$

If $Z$ is a compact space, by the suspension of $Z$ we will mean the space $\Sigma Z=(Z \times[-1,1]) /\{Z \times-1, Z \times 1\}$. For $r \geqq 1$, the $r$ th suspension of $Z$ is defined inductively by $\Sigma^{r} Z=\Sigma\left(\Sigma^{r-1} Z\right)$, where $\Sigma^{0} Z=Z$ and $\Sigma^{1} Z=\Sigma Z$. If $\tilde{Z}$ and $Z$ are two compact spaces, and $h: \tilde{Z} \rightarrow Z$ is a continuous map (= map), then $\Sigma h: \Sigma \tilde{Z} \rightarrow \Sigma Z$ is the map defined by

$$
\Sigma h(\tilde{\rho}(z, t))=\rho(h(z), t)
$$


where $\tilde{\rho}: \tilde{Z} \times[-1,1] \rightarrow \Sigma \tilde{Z}$ and $\rho: Z \times[-1,1] \rightarrow \Sigma Z$ are the quotient maps. Inductively, $\Sigma^{r} h: \Sigma^{r} \tilde{Z} \rightarrow \Sigma^{r} Z$ is defined by $\Sigma^{r} h=\Sigma\left(\Sigma^{r-1} h\right)$ for $r \geqq 2$.

If $K^{p}$ is a finite complex, $A^{t}=b * B^{t-1}$ is a $t$-simplex of $K^{p}$ (where $b$ is a vertex of $A^{t}, B^{t-1}$ is the face of $A^{t}$ opposite to $b$, and $*$ denotes join), and $\widetilde{K}^{p}$ is a subcomplex of $K^{p}$ such that $K^{p}=\widetilde{K}^{p}+A^{t}+B^{t-1}\left(\left|K^{p}\right|=\left|\widetilde{K}^{p}\right| \cup\left|A^{t}\right|\right)$ and $\tilde{K}^{p} \cap A^{t}$ $=b * B^{t-1}$, then the move $K^{p} \searrow \widetilde{K}^{p}$ is called an elementary collapse of $K^{p}$ to $\widetilde{K}^{p}$. If $L^{p} \subset K^{p}$ and there exists a finite sequence of elementary collapses $K^{p} \searrow \widetilde{K}_{1}^{p}$ $\searrow \widetilde{K}_{2}^{p} \searrow \cdots \widetilde{K}_{n}^{p} \searrow L^{p}$, we say $K^{p}$ collapses to $L^{p}$. If $K^{p}$ collapses to a vertex, we say $K^{p}$ is collapsible.

If $X$ is a topological space, we will call $G$ a decomposition of $X$ if $G$ is a collection of mutually exclusive subsets of $X$ whose union is $X$. By $X / G$ we will mean the topological space whose points are elements of $G$ and whose topology is given as follows: Let $\rho: X \rightarrow X / G$ be the function defined by $\rho(x)=g$, where $g$ is the unique element of $G$ containing the point $x$. The topology of $X / G$ is defined by $V \subset X / G$ is open if and only if $\rho^{-1}(V)$ is open in $X$. (Thus, if $h: X \rightarrow Y$ is a continuous map and $G=\left\{h^{-1}(y) \mid y \in Y\right\}$, then $h \circ \rho^{-1}: X / G \rightarrow Y$ is a 1-1 continuous map of $X / G$ into $Y$.)

If $D$ is a $k$-cell in $E^{m}$, we say $D$ is locally flat in $E^{m}$ if for every $x \in \operatorname{Int} D(x \in \operatorname{Bd} D)$ there exists an open set $U$ in $E^{m}$ containing $x$ such that $(U, U \cap D) \approx\left(E^{m}, E^{k}\right)$ $\left((U, U \cap D) \approx\left(E^{m}, E_{+}^{k}\right)\right)$. If $Z$ is a subset of $E^{m}$, we will say $E^{m}-Z$ is $1-\mathrm{LC}$ at each point $z \in Z$, if given $z \in Z$ and $U$ open in $E^{m}$ containing $z$, then there exists an open subset $V \subset E^{m}$ containing $z$ such that $V \subset U$ and each loop in $V-Z$ is inessential in $U-Z$. Finally, by a pseudo-isotopy $f_{t}(t \in[0,1])$ carrying a space $X$ onto itself, we will mean a continuous function $f: X \times[0,1] \rightarrow X$ such that the function $f_{t}: X \rightarrow X$, defined by $f_{t}(x)=f(x, t)$, is a homeomorphism carrying $X$ onto itself for each $t \in[0,1)$.

I would like to thank the referee for many helpful criticisms.

1. The key isotopy theorem and the main results. The key isotopy theorem on which all our results are based is as follows:

THEOREM 1.1. Suppose $F^{p}$ is a p-cell embedded as a subset of a separable metric space $X^{q}$, where $X^{q} \times E^{r} \approx E^{q+r}$. If $r \geqq 1$ and $F^{p}$ is $(r-1)$-flat in $X^{q} \times E^{r}$ (e.g., if $q-p \neq 1$ and $q+r \geqq 5)$, then, given $\varepsilon>0$, there exists an isotopy $\mu_{t}(t \in[0,1])$ of $X^{q} \times E^{r}$ onto itself such that

(1) $\mu_{0}=$ identity;

(2) $\mu_{t} \mid\left(X^{q} \times E^{r}\right)-\left(U_{\varepsilon}\left(F^{p}, X^{q}\right) \times E^{r}\right)=$ identity, for each $t \in[0,1]$;

(3) if $(x, w) \in X^{q} \times E^{r}$ and $\mathrm{pr}_{2}: X^{q} \times E^{r} \rightarrow E^{r}$ is the projection onto $E^{r}$, then $\left\|w-\operatorname{pr}_{2}\left(\mu_{t}(x, w)\right)\right\|<\varepsilon$, for each $t \in[0,1]$;

(4) $\mu_{1}$ is uniformly continuous; and

(5) if $w \in E^{r}$, then diam $\mu_{1}\left(F^{p} \times w\right)<\varepsilon$.

This theorem will be obtained via two reductions. The first is given in $\S 2$ and the second is given in $\$ 3$. The second reduction follows directly from the work and 
results of Bryant in [3]. Since a simple extension of this work will easily include our additional requirements here, we will only just briefly discuss in $\$ 3$ some of the pertinent facts and results related to [3]. In $\S 2$, we show that our modification of Bryant's work allows us to obtain the above key isotopy theorem. In this section, we apply Theorem 1.1 and obtain all our main results.

CoROllaRY 1.2. Suppose $F^{p}$ is a p-cell embedded as a subset of a separable metric space $X^{q}$, where $X^{q} \times E^{r} \approx E^{q+r}$. Let $G$ denote the decomposition of $X^{q} \times E^{r}$ defined by

$$
G=\left\{g \subset X^{q} \times E^{r} \mid g=F^{p} \times w, \text { or } g=(x, w), x \in X^{q}-F^{p}, w \in E^{r}\right\} .
$$

If $r \geqq 1$ and $F^{p}$ is $(r-1)$-flat in $X^{q} \times E^{r}$, then, given $\varepsilon>0$, there is a pseudo-isotopy $f((x, w), t)\left((x, w) \in X^{q} \times E^{r}, 0 \leqq t \leqq 1\right)$ of $X^{q} \times E^{r}$ onto itself such that

(1) $f((x, w), 0)$ is the identity (i.e., $f((x, w), 0)=(x, w))$;

(2) if $(x, w) \in\left(X^{q} \times E^{r}\right)-\left(U_{\varepsilon}\left(F^{p}, X^{q}\right) \times E^{r}\right)$, then $f((x, w), t)=(x, w)$, for each $t \in[0,1]$;

(3) if $(x, w) \in X^{q} \times E^{r}$ and $\mathrm{pr}_{2}: X^{q} \times E^{r} \rightarrow E^{r}$ is the projection onto $E^{r}$, then $\left\|w-\operatorname{pr}_{2}(f((x, w), t))\right\|<\varepsilon$, for each $t \in[0,1]$;

(4) $f((x, w), 1)$ takes $X^{q} \times E^{r}$ onto itself and each element of $G$ onto a distinct point of $X^{q} \times E^{r}$.

Proof. This follows as in the proof of Theorem 3 of [2]. The pseudo-isotopy is obtained simply by a sequence of applications of Theorem 1.1. For convenience, we will state the appropriate inductive step here. The remainder of the proof should be clear from this. Refer to [2] for further details.

We first note that the following conclusion can also be added to Theorem 1.1:

(5) There exists a $\delta>0$, such that if $w \in E^{r}$, then diam $\mu_{1}\left(U_{\delta}\left(F^{p}, X^{q}\right) \times w\right)<\varepsilon$.

That is, given $\varepsilon>0$, suppose $\mu_{t}$ is an isotopy satisfying Theorem 1.1 , where the $\varepsilon>0$ we use there is $\varepsilon / 2$. By conclusion (4), if $w \in E^{k}$, then diam $\mu_{1}\left(F^{p} \times w\right)<\varepsilon / 2$. Since $\mu_{1}$ is uniformly continuous, there exists a $\delta>0$ such that if $Z$ is a set in $X^{q} \times E^{r}$ of diameter $<\delta$, then $\operatorname{diam} \mu_{1}(Z)<\varepsilon / 4$. It follows then that

$$
\operatorname{diam} \mu_{1}\left(U_{\delta}\left(F^{p}, X^{q}\right) \times w\right)<\varepsilon .
$$

Let $\varepsilon_{1}, \varepsilon_{2}, \ldots$ be a sequence of positive numbers such that $2 \sum_{i=1}^{\infty} \varepsilon_{i}<\varepsilon$. By induction, using Theorem 1.1, we define a monotone decreasing sequence $\delta_{1}<\varepsilon$, $\delta_{2}, \ldots$ of positive numbers tending to zero and a sequence of isotopies

$$
\begin{array}{ll}
f((x, w), t) & \left((x, w) \in X^{q} \times E^{r}, 0 \leqq t \leqq \frac{1}{2}\right), \\
f((x, w), t) & \left((x, w) \in X^{q} \times E^{r}, \frac{1}{2} \leqq t \leqq \frac{2}{3}\right), \ldots
\end{array}
$$

such that $f((x, w), 0)=(x, w)$, two adjacent $f(x, t)$ 's agree on their common end, each $f((x, w), i /(i+1))$ is uniformly continuous,

(1) $f((x, w),(i-1) / i)=f((x, w), t) \quad((i-1) / i \leqq t \leqq i /(i+1))$ except possibly on $U_{\delta_{i}}\left(F^{p}, X^{q}\right) \times E^{r}$, 
(2) $\operatorname{diam} f\left(U_{\delta_{i+1}}\left(F^{p}, X^{q}\right) \times w, i /(i+1)\right)<\varepsilon_{i}, w \in E^{r}$,

(3) no point moves more than $2 \varepsilon_{i-1}$ during $f(x, t)((i-1) / i \leqq t \leqq i /(i+1))$, and

(4) $f\left(X^{q} \times U_{\varepsilon_{i}}\left(w, E^{r}\right),(i-1) / i\right) \supset f\left(X^{q} \times w, i /(i+1)\right)$.

The proof is then completed by noting $(\mathrm{A})$ that the existence of such a sequence follows easily from Theorem $1.1(f((x, w), t)((i-1) / i \leqq t \leqq i /(i+1))$ is of the form $f\left(\mu_{t}^{i}(x, w),(i-1) / i\right)$, where $\mu_{t}^{i}(x, w) \quad((i-1) / i \leqq t \leqq i /(i+1))$ is an appropriately chosen $\mu_{t}$ from Theorem 1.1) and (B) that the existence of such a sequence is enough to guarantee the truth of Corollary 1.2.

Corollary 1.3. Suppose $F^{p}$ is a p-cell embedded as a subset of a separable metric space $X^{q}$, where $X^{q} \times E^{r} \approx E^{q+r}$. Let $G$ denote the decomposition of $X^{q} \times E^{r}$ as in Corollary 1.2, and let $\rho: X^{q} \times E^{r} \rightarrow\left(X^{q} \times E^{r}\right) / G=\left(X^{q} / F^{p}\right) \times E^{r}$ denote the quotient map (if $\rho^{\prime}: X^{q} \rightarrow X^{q} / F^{p}$ is the quotient map, then $\rho=\rho^{\prime} \times \mathrm{id}_{E^{r}}$ ). If $r \geqq 1$ and $F^{p}$ is $(r-1)$-flat in $X^{q} \times E^{r}$, then given $\varepsilon>0$ there exists a homeomorphism

$$
h:\left(X^{q} / F^{p}\right) \times E^{r} \rightarrow X^{q} \times E^{r}\left(\approx E^{q+r}\right)
$$

such that $h \circ \rho=$ identity on $\left\{X^{q}-U_{\varepsilon}\left(F^{p}, X^{q}\right)\right\} \times E^{r}$ and $h$ is bounded by $\varepsilon$ on the $E^{r}$ factor (as in (3) of Corollary 1.2).

Proof. By Corollary 1.3, there exists a map $f_{1}: X^{q} \times E^{r} \rightarrow X^{q} \times E^{r}$ such that $f_{1}=$ identity outside $U_{\varepsilon}\left(F^{p}, X^{q}\right) \times E^{r}$,

for each $(x, w) \in X^{q} \times E^{r},\left\|w-\mathrm{pr}_{2} \circ f_{1}(x, w)\right\|<\varepsilon$, and

$\left\{f_{1}^{-1}(x, w) \mid(x, w) \in X^{q} \times E^{r}\right\}=G$.

Let $h:\left(X^{q} / F^{p}\right) \times E^{r} \rightarrow X^{q} \times E^{r}$ be the 1-1 continuous map induced by the following diagram:

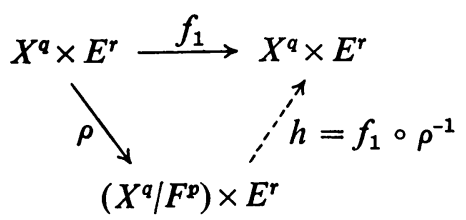

Since $X^{q} \times E^{r} \approx E^{q+r}$ and $f_{1}$ is a compact map (i.e., preimages of compact sets are compact), $\left(X^{q} / F^{p}\right) \times E^{r} \approx E^{q+r}$ and $h$ is a homeomorphism. Clearly, the remaining properties are satisfied.

Lemma 1.4. Given integers $0 \leqq u<v \leqq w$, suppose that $D$ is a $v$-cell in $E^{w}(w \geqq 4)$, $P$ is a finite $u$-dimensional polyhedron in $\mathrm{Bd} D(w-u \geqq 4)$, such that each open simplex of $P$ is locally flat in $\mathrm{Bd} D, D-P$ is locally flat in $E^{w}$, and $E^{w}-P$ is 1-LC at each point of $P$. Then $D$ is flat.

The result is proven in [5, Theorem 5.2], for $w \geqq 5$, and in [15, Corollary 2.5], for $u=0$ and $w \geqq 4$.

LemmA 1.5. Suppose $A$ is a t-cell topologically embedded as a subset of a separable metric space $X^{q}$, where $X^{q} \times E^{r} \approx E^{q+r}$, and $B$ is a $(t-1)$-cell in $\mathrm{Bd} A$ such that 
$(A, B) \approx\left(\Delta^{t}, \Delta^{t-1}\right)$, where $\Delta^{t}$ is a $t$-simplex and $\Delta^{t-1}$ is a $(t-1)$-dimensional face of $\Delta^{t}$. Also, suppose $Z$ is a compact subset of $X^{q}$ such that $\left(X^{q} / Z\right) \times E^{r} \approx E^{q+r}$ and $Z \cap A=\operatorname{Bd} A$-Int $B=C$. If $q \geqq 3, r \geqq 1,0 \leqq k \leqq r-1$, and $A$ is $k$-flat in $X^{q} \times E^{r}$, then $A / C$ is a $t$-cell in $X^{q} / Z$ such that $A / C$ is $k$-flat in $\left(X^{q} / Z\right) \times E^{r}$.

Proof. Since $Z \cap A=C$, and $(A, C) \approx\left(\Delta^{t}, v * \Delta^{t-1}\right)$, it is clear that $\rho(A \cup Z)$ $=(A \cup Z) / Z=A / C=\tilde{D}$ is a $t$-cell in $X^{q} / Z$, where $\rho: X^{q} \rightarrow X^{q} / Z$ is the quotient map. Since $A$ is $k$-flat in $X^{q} \times E^{r}$,

$$
\left(E^{q+r}, I^{t+k}\right) \approx\left(X^{q} \times E^{r}, A \times I^{k}\right)=\left(X^{q} \times E^{k} \times E^{r-k}, A \times I^{k} \times \theta\right),
$$

where $\theta$ denotes the origin of $E^{r-k}$ and $r-k \geqq 1$.

Under the quotient map $\rho$, this latter set is carried to

$$
\left(\left(X^{q} / Z\right) \times E^{k} \times E^{r-k},(A / C) \times I^{k} \times \theta\right) .
$$

Let $D=(A / C) \times I^{k}$ and $P=\{C\} \times I^{k} \subset \mathrm{Bd} D$. Then $D$ is a $(t+k)$-cell and $P$ is a $k$-cell in $\mathrm{Bd} D$ that is flat in $\mathrm{Bd} D$. Since $\rho$ is a homeomorphism outside of $Z \times E^{r}$, and $\left(Z \times E^{r}\right) \cap\left(A \times I^{k}\right)=C \times I^{k}$, it follows that $D-P$ is locally flat in $\left(X^{q} / Z\right) \times E^{r} \approx E^{q+r}$. Since $P \subset\left(X^{q} / Z\right) \times E^{k} \times \theta \subset\left(X^{q} / Z\right) \times E^{k} \times E^{r-k} \approx E^{q+r},(q+k)$ $-k \geqq 3$, and $r-k \geqq 1$, it follows easily that $\left(\left(X^{q} / Z\right) \times E^{r}\right)-P$ is $1-\mathrm{LC}$ at each point of $P$. Let $w=q+r, v=t+k$, and $u=k$. Since $q \geqq 3, r \geqq 1, r-k \geqq 1$, and $t \leqq q$, the result follows from Lemma 1.4 , since $w \geqq 4,0 \leqq u<v<w$ (we may suppose $t>0$, for the result is trivial for $t=0$ ), and $w-u \geqq 4$.

THEOREM 1.6. Suppose $K^{p}$ is a finite collapsible p-complex embedded as a subset of a separable metric space $X^{q}$, where $X^{q} \times E^{r} \approx E^{q+r}$. Let $G\left(X^{q}, K^{p}, r\right)$ denote the decomposition of $X^{q} \times E^{r}$ defined by

$$
\begin{aligned}
& G\left(X^{q}, K^{p}, r\right)=\left\{g \subset X^{q} \times E^{r} \mid g=K^{p} \times w,\right. \text { or } \\
& \left.\quad g=(x, w), x \in X^{q}-K^{p}, w \in E^{r}\right\},
\end{aligned}
$$

and let $\rho: X^{q} \times E^{r} \rightarrow\left(X^{q} \times E^{r}\right) / G\left(X^{q}, K^{p}, r\right)=\left(X^{q} / K^{p}\right) \times E^{r}$ denote the quotient map. If $r \geqq 1, q \geqq 3$, and each simplex of $K^{p}$ is $(r-1)$-flat in $X^{q} \times E^{r}$, then, given $\varepsilon>0$, there exists a homeomorphism

$$
h:\left(X^{q} / K^{p}\right) \times E^{r} \rightarrow X^{q} \times E^{r} \approx E^{q+r}
$$

such that $h \circ \rho=$ identity on $\left\{X^{q}-U_{\varepsilon}\left(K^{p}, X^{q}\right)\right\} \times E^{r}$ and, if $(\tilde{x}, w) \in\left(X^{q} / K^{p}\right) \times E^{r}$ and $\mathrm{pr}_{2}: X^{q} \times E^{r} \rightarrow E^{r}$ is the projection onto $E^{r}$, then

$$
\left\|w-\operatorname{pr}_{2}(h(\tilde{x}, w))\right\|<\varepsilon
$$

Proof. If $K^{p}$ is a $p$-simplex (or is merely homeomorphic to a $p$-simplex), then the corollary follows directly from Corollary 1.3. Otherwise, the proof will be by induction on the number of elementary collapses, say $\nu\left(K^{p}\right)$, required to collapse $K^{p}$ to a point. By the first remark, we have the result for $v\left(K^{p}\right)=1$. Hence, suppose inductively, for some $k \geqq 1$, we know the result for all pairs $\left(\widetilde{X}^{q}, \widetilde{K}^{p}\right)$ satisfying the hypothesis of Theorem 1.6 , where $\nu\left(\widetilde{K}^{p}\right) \leqq k$. 
Suppose we are given $\varepsilon>0$, and $K^{p} \subset X^{q}$ is a finite collapsible $p$-complex such that $\nu\left(K^{p}\right)=k+1$, where the pair satisfies the hypothesis of Theorem 1.6. Let $K^{p} \searrow \widetilde{K}^{s}$ be the first collapse in the sequence which collapses $K^{p}$ to a point. Then $K^{p}=\tilde{K}^{s}+A^{t}$, where $A^{t}=b * B^{t-1}, \tilde{K}^{s} \cap A^{t}=b * B^{t-1}, 1 \leqq s \leqq p$, and $1 \leqq t \leqq p$. By induction, the result holds for $\widetilde{K}^{s} \subset X^{q}$.

That is, there exists a homeomorphism

$$
\hat{h}:\left(X^{q} / \tilde{K}^{s}\right) \times E^{r} \rightarrow X^{q} \times E^{r}
$$

such that $\hat{h} \circ \hat{\rho}=$ identity on $\left\{X^{q}-U_{\varepsilon / 2}\left(\tilde{K}^{s}, X^{q}\right)\right\} \times E^{r}$ and $\hat{h}$ is bounded by $\varepsilon / 2$ on the $E^{r}$ factor. Let $\tilde{X}^{q}=\left(X^{q} / \tilde{K}^{s}\right)$ and let $\tilde{A}^{t}$ denote the $t$-cell in $\tilde{X}^{q}$ given by $\tilde{A}^{t}=\hat{\rho}\left(K^{p}\right)$ $=K^{p} / \tilde{K}^{s}=A^{t} / b * B^{t-1}$. Since $\hat{h}$ is a homeomorphism and $X^{q} \times E^{r} \approx E^{q+r}, \tilde{X}^{q} \times E^{r}$ $\approx E^{q+r}$ and $\tilde{X}^{q}$ is a separable metric space. Thus, if $K^{p} \subset X^{q}$ satisfies the given hypothesis, then $\tilde{A}^{t} \subset\left(K^{q} / \tilde{K}^{s}\right)$ also satisfies the given hypothesis. That is, since $A^{t}$ is $(r-1)$-flat in $X^{q} \times E^{r}$, it follows by Lemma 1.5 that $\tilde{A}^{t}$ is $(r-1)$-flat in $\left(X^{q} / \tilde{K}^{s}\right) \times E^{r}$. Therefore, we have that $\tilde{A}^{t} \subset \tilde{X}^{q}$ satisfies the hypothesis of Corollary 1.3.

Let $\delta$ denote a positive number such that

$$
\delta<\varepsilon / 2 \text { and }\left(\hat{\rho}^{\prime}\right)^{-1}\left(U_{\delta}\left(\tilde{A}^{t}, \tilde{X}^{q}\right)\right) \subset U_{\varepsilon / 2}\left(K^{p}, X^{q}\right),
$$

where $\hat{\rho}^{\prime}: X^{q} \rightarrow X^{q} / \tilde{K}^{s}=\tilde{X}^{q}$ is the quotient map $\left(\hat{\rho}=\hat{\rho}^{\prime} \times \mathrm{id}_{E^{r}}\right)$. By Corollary 1.3, there exists a homeomorphism

$$
\tilde{h}:\left(\tilde{X}^{q} / \tilde{A}^{t}\right) \times E^{r} \rightarrow \tilde{X}^{q} \times E^{r}
$$

such that $\tilde{h} \circ \tilde{\rho}=$ identity on $\left\{\tilde{X}^{q}-U_{\delta}\left(\tilde{A}^{t}, \tilde{X}^{q}\right)\right\} \times E^{r} \supset \hat{\rho}\left(\left\{X^{q}-U_{\varepsilon_{i} 2}\left(K^{p}, X^{q}\right)\right\} \times E^{r}\right.$ and $\tilde{h}$ is bounded by $\delta$ on the $E^{r}$ factor.

We claim that $\hat{h} \circ \tilde{h} \circ \tilde{\rho} \circ \hat{\rho}=$ identity on $\left\{X^{q}-U_{\varepsilon / 2}\left(K^{p}, X^{q}\right)\right\} \times E^{r}=W$. Since $\hat{\rho}(W) \subset\left\{\tilde{X}^{q}-U_{\delta}\left(\tilde{A}^{t}, \tilde{X}^{q}\right)\right\} \times E^{r}$ and $\tilde{h} \circ \tilde{\rho}=$ identity on this latter set, $\tilde{h} \circ \tilde{\rho} \circ \hat{\rho} \mid W$ $=\hat{\rho} \mid W$. Since $\left\{X^{q}-U_{\varepsilon / 2}\left(\widetilde{K}^{s}, X^{q}\right)\right\} \times E^{r} \supset W$ and $\hat{h} \circ \hat{\rho}=$ identity on this former set, it follows that identity $=\hat{h} \circ \hat{\rho}|W=\hat{h} \circ \tilde{h} \circ \tilde{\rho} \circ \hat{\rho}| W$.

Let $h^{\prime}$ denote the 1-1 continuous map induced by the following diagram:

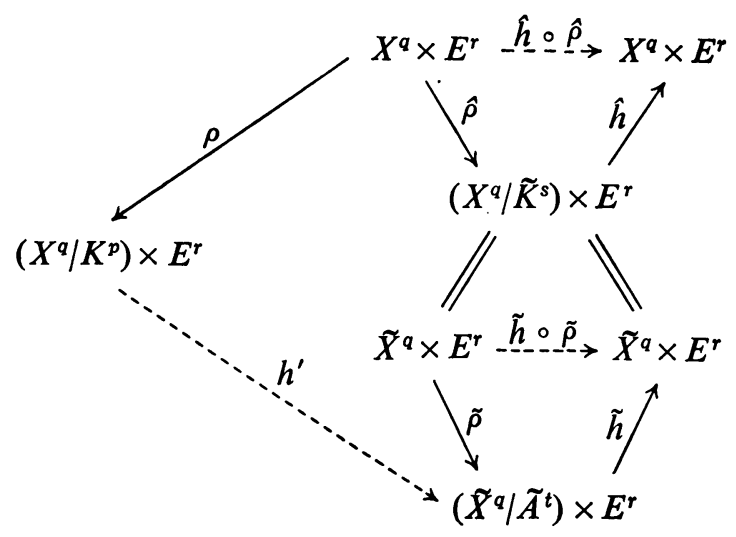


That is, since $\left\{(\tilde{\rho} \circ \hat{\rho})^{-1}(\tilde{x}, w) \mid \tilde{x} \in\left(\tilde{X}^{q} / \tilde{A}^{t}\right), w \in E^{r}\right\}=G,(\tilde{\rho} \circ \hat{\rho}) \circ \rho^{-1}=h^{\prime}$ is a $1-1$ continuous map carrying $\left(X^{q} / K^{p}\right) \times E^{r}$ onto $\left(\tilde{X}^{q} / \tilde{A}^{t}\right) \times E^{r}$. Since $(\tilde{\rho} \circ \hat{\rho})$ is a compact map, $h^{\prime}$ is a homeomorphism.

Let $h:\left(X^{q} / K^{p}\right) \times E^{r} \rightarrow X^{q} \times E^{r}$ be the homeomorphism defined by $h=\hat{h} \circ \tilde{h} \circ h^{\prime}$. Since $h^{\prime}$ does not change $E^{r}$ coordinates, $\tilde{h}$ changes $E^{r}$ coordinates $<\delta<\varepsilon / 2$, and $\hat{h}$ changes $E^{r}$ coordinates $<\varepsilon / 2$, it follows that $h$ changes $E^{r}$ coordinates $<\varepsilon$. Finally, we claim $h \circ \rho=$ identity on $\left\{X^{q}-U_{\varepsilon / 2}\left(K^{p}, X^{q}\right)\right\} \times E^{r}=W$. This follows since $h \circ \rho=\left(\hat{h} \circ \tilde{h} \circ h^{\prime}\right) \circ \rho=\hat{h} \circ \tilde{h} \circ\left[(\tilde{\rho} \circ \hat{\rho}) \circ \rho^{-1}\right] \circ \rho=\hat{h} \circ \tilde{h} \circ \tilde{\rho} \circ \hat{\rho}$, and we noted above that $\hat{h} \circ \tilde{h} \circ \tilde{\rho} \circ \hat{\rho} \mid W=$ identity. This completes the proof of Theorem 1.6.

COROLlaRY 1.7. If $K^{p}$ is a finite collapsible p-complex topologically embedded in $E^{n}$ and either $n=3$ and $p \leqq 3$, or $n \geqq 4$ and $p \leqq n-2$, then $\left(E^{n} / K^{p}\right) \times E^{1} \approx E^{n+1}$.

Proof. If $n=3$ or $n \geqq 4$, it follows by [11] or [6], respectively, that each simplex of $K^{p}$ is flat in $E^{n+1}$. Thus, in either case, the result follows from Theorem 1.6.

REMARK. As we noted earlier, in [4], Bryant shows that if $D$ is an $(n-1)$-cell in $E^{n}(n \geqq 4)$, then $\left(E^{n} / D\right) \times E^{1} \approx E^{n+1}$. In fact, he shows that Theorem 1.1 is valid for $q \geqq 4, p=q-1, r=1$, and $X^{q}=E^{q}$. This result appears to hold for $F^{q-1} \subset X^{q}$, where $X^{q} \times E^{1} \approx E^{q+1}$ and $q \geqq 4$. (Actually, we just have to consider the case where $X^{q}=E^{q} /\{$ a collapsible $p$-complex $\}$.) If this is the case, then Corollary 1.7 holds for $n \geqq 3$ and all $p \leqq n$. Recall, by [7], Theorem 1.1 is valid for $F^{q} \subset X^{q}$, where $X^{q} \times E^{1}$ $\approx E^{q+1}$ and $q \geqq 4$.

Corollary 1.8. Suppose $K^{p}$ is a finite collapsible p-complex embedded as a subset of a separable metric space $X^{q}$, where $X^{q} \times E^{r} \approx E^{q+r}$. Also, suppose that $Z^{q}$ is a compact q-dimensional metric space containing $X^{q}, \hat{G}$ is the decomposition of $Z^{q}$ given by $\hat{G}=\left\{\hat{g} \subset Z^{q} \mid \hat{g}=K^{p}\right.$ or $\left.\hat{g}=x \in Z^{q}-K^{p}\right\}$, and $\hat{\rho}: Z^{q} \rightarrow Z^{q} / \hat{G}=Z^{q} / K^{p}$ is the quotient map. If $r \geqq 1, q \geqq 3$, and each simplex of $K^{p}$ is $(r-1)$-flat in $X^{q} \times E^{r}$, then, given $\varepsilon>0$,

(A) there exists a homeomorphism $h_{1}: \Sigma^{r}\left(Z^{q} / K^{p}\right) \rightarrow \Sigma^{r}\left(Z^{q}\right)$ such that $h_{1} \circ \Sigma^{r} \hat{\rho}$ $=$ identity on $\Sigma^{r}\left(Z^{q}-U_{\varepsilon}\left(K^{p}, X^{q}\right)\right)$.

Furthermore, if $Z^{q}$ contains a topological $q$-cell $\hat{B}^{q}$ such that $\left(Z^{q}-\operatorname{Int} \hat{B}^{q}\right) \subset X^{q}$, where $q+r \geqq 5$ and $q \geqq 3$, then

(B) there exists a homeomorphism $h_{2}: \Sigma^{r}\left(Z^{q} / K^{p}\right) \rightarrow \Sigma^{r}\left(S^{q}\right) \approx S^{q+r}$ and a flat $q$-cell $B^{q} \subset\left(\right.$ Int $\left.\hat{B}^{q}\right)-K^{p}$ such that $h_{2} \circ \Sigma^{r} \hat{\rho}=$ identity on $\Sigma^{r} B^{q}$, where $B^{q}$ is also identified with a flat q-cell in $S^{q}$.

Before we give the proof of Corollary 1.8, we first make some elementary observations. If $Z$ is a compact $q$-dimensional metric space, then we can think of $\Sigma^{r} Z$ as $Z * S^{r-1} \subset E^{m} \times E^{r}$, where $Z \subset E^{m}=\theta_{r}$ and $S^{r-1} \subset \theta_{m} \times E^{r}\left(\theta_{r}\right.$ and $\theta_{m}$ denote the origin of $E^{r}$ and $E^{m}$, respectively). Moreover, every point of $\left(Z * S^{r-1}\right)-S^{r-1}$ has a unique representation in the form $\langle z, t y\rangle$, where $z \in Z, y \in S^{r-1}$ and $t \in[0,1)$ (i.e., $\left.\langle z, t y\rangle=(1-t) z+t y \in z * y \subset Z * S^{r-1}\right)$. Let $\Phi_{z}^{r}$ be the homeomorphism carrying $Z \times E^{r}$ onto $\left(Z * S^{r-1}\right)-S^{r-1}$ defined by sending $(z, w) \in Z \times E^{r}$ to

$$
\langle z,(\|w\| / 1+\|w\|)(w /\|w\|)\rangle \quad(\text { if }\|w\| \neq 0)
$$


and sending $(z, 0)$ to

$$
\langle z, 0\rangle=z \in Z \subset Z * S^{r-1} .
$$

(1.7.1) Thus, if $h: \tilde{Z} \times E^{r} \rightarrow Z \times E^{r}$ is a continuous map of $\tilde{Z} \times E^{r}$ onto $Z \times E^{r}$ that is bounded on the $E^{r}$ factor, then $h$ induces a continuous map $\tilde{h}: \Sigma^{r} \tilde{Z} \rightarrow \Sigma^{r} Z$ as follows:

If $h^{\prime}: \tilde{Z} * S^{r-1} \rightarrow Z * S^{r-1}$ is the map defined by $h^{\prime}=$ identity on $S^{r-1}$ and $h^{\prime}=\Phi_{Z}^{r} \circ \tilde{h} \circ\left(\Phi_{Z}^{r}\right)^{-1}$ on $\left(\tilde{Z} * S^{r-1}\right)-S^{r-1}$, then $\tilde{h}$ is the composition

$$
\Sigma^{r} \tilde{Z} \longrightarrow \tilde{Z} * S^{r-1} \stackrel{h^{\prime}}{\longrightarrow} Z * S^{r-1} \longrightarrow \Sigma^{r} Z \text {. }
$$

(1.7.2) Furthermore, if $\tilde{A} \subset \tilde{Z}, A \subset Z$, and $h \mid \tilde{A} \times E^{r}: \tilde{A} \times E^{r} \rightarrow A \times E^{r}$ is of the form $\hat{h} \times \operatorname{id}_{E^{r}}$ where $\hat{h}: \tilde{A} \rightarrow A$, then $\tilde{h}$ (defined as above) $=\Sigma^{r} \hat{h}$ on $\Sigma^{r-1} \tilde{A} \subset \Sigma^{r-1} \tilde{Z}$. These observations are proven as an elementary lemma in [9].

One additional result that we will use here is Theorem III.3.3, (3) of [10], where the author proved the following:

(1.7.3) Suppose $Z$ is a compact connected metric space such that $Z$ is simply connected, contains a $q$-cell $N^{q}$, and has the same homology type as the $q$-sphere. Let $C^{q}$ denote a flat $q$-cell in Int $N^{q}, T^{r}=S^{1} \times \cdots \times S^{1}, r$-times, and consider the $q$-sphere $S^{q}$ as the natural union of the $q$-cell $C^{q}$ and another $q$-cell $B^{q}$. If $Z \times E^{r}$ is an open $(q+r)$-manifold, where $q+r \geqq 5$ and $q>2$, and $D^{q}=Z-\operatorname{Int} C^{q}$, then there exist homeomorphisms

$$
\begin{aligned}
& h_{1}: D^{q} \times T^{r} \rightarrow B^{q} \times T^{r}, \quad h_{2}: D^{q} \times E^{r} \rightarrow B^{q} \times E^{r}, \\
& h_{3}: \Sigma^{r} D^{q} \rightarrow \Sigma^{r} B^{q}, \quad \text { and } \quad h_{4}: \Sigma^{r} Z \rightarrow \Sigma^{r} S^{q} .
\end{aligned}
$$

The homeomorphism $h_{1}$ is the identity on $\mathrm{Bd} D^{q} \times T^{r}=\mathrm{Bd} C^{q} \times T^{r}$. The homeomorphism $h_{2}$ is the natural covering of $h_{1}$, so as to be the identity on $\mathrm{Bd} D^{q} \times E^{r}$, and is bounded on the $E^{r}$ factor. The homeomorphism $h_{3}$ is induced from $h_{2}$, as above, and is the identity on the suspension $(r-1)$-sphere. The homeomorphism $h_{4}$ is obtained by extending $h_{3}$ by the identity homeomorphism on $\Sigma^{r} C^{q}$.

Proof. By Theorem 1.6, there exists a homeomorphism

$$
h:\left(X^{q} / K^{p}\right) \times E^{r} \rightarrow X^{q} \times E^{r} \approx E^{q+r}
$$

such that $h \circ \rho=$ identity on $\left\{X^{q}-U_{\varepsilon}\left(K^{p}, X^{q}\right)\right\} \times E^{r}$ and, if $(\tilde{x}, w) \in\left(X^{q} / K^{p}\right) \times E^{r}$ and $\operatorname{pr}_{2}: X^{q} \times E^{r} \rightarrow E^{r}$ is the projection onto $E^{r}$, then $\left\|w-\operatorname{pr}_{2} \circ h(\tilde{x}, w)\right\|<\varepsilon$. Conclusion (A) follows immediately from this result, (1.7.1), and (1.7.2).

Conclusion (B) follows from Conclusion (A) and (1.7.3). That is, since $X^{q} \times E^{r} \approx E^{q+r}, \pi_{i}\left(X^{q}\right)=0$ for all $i \geqq 1$. Since $Z^{q}=X^{q} \cup B^{q}, Z^{q}$ has the same homology groups as $S^{q}$ and $Z^{q}$ is simple connected. Clearly, $Z^{q} \times E^{r}$ is an open $(q+r)$-manifold. Since $K^{p}$ is a compact subset of $X^{q}$, there exists a flat $q$-cell $B^{q} \subset$ Int $\hat{B}^{q}$ such that $B^{q} \cap K^{p}=\varnothing$. By (1.7.3) (with $C^{q}$ of (1.7.3) corresponding to $B^{q}$ here), $\Sigma^{r} Z^{q} \approx \Sigma^{r} S^{q}$ by a homeomorphism that is the identity on $\Sigma^{r} B^{q}$. If we 
denote this latter homeomorphism by $\tilde{h}$ and $\varepsilon>0$ is so small that $B^{q} \cap U_{\varepsilon}\left(K^{p}, X^{q}\right)$ $=\varnothing$, then Conclusion (B) follows by defining $h_{2}=\tilde{h} \circ h_{1}$.

THEOREM 1.9. Suppose $K^{p}$ is a finite collapsible p-complex embedded as a subset of a separable metric space $X^{q}$, where $X^{q} \times E^{r} \approx E^{q+r}$. Let $s$ be an integer such that $0 \leqq s \leqq r-1$ and let $E^{r}=E^{s} \times E^{r-s}\left(E^{0} \times E^{r-0}=E^{r}\right)$. Also, suppose $L^{t}$ is a finite collapsible $t$-complex embedded as a subset of $E^{s}$, where $0 \leqq t \leqq s$ (if $s=0, K^{p} \times L^{0}$ $\left.=K^{p}\right)$. If $q+r \geqq 5, r \geqq 1$, and $(q+s)-(p+t) \geqq 2$, then, given $\varepsilon>0$, there exists $a$ homeomorphism

$$
h:\left\{\left(X^{q} \times E^{s}\right) /\left(K^{p} \times L^{t}\right)\right\} \times E^{r-s} \rightarrow\left(X^{q} \times E^{s}\right) \times E^{r-s} \approx E^{q+r}
$$

such that, if $\rho:\left(X^{q} \times E^{s}\right) \times E^{r-s} \rightarrow\left\{\left(X^{q} \times E^{s}\right) /\left(K^{p} \times L^{t}\right)\right\} \times E^{r-s}$ is the quotient map, then $h \circ \rho=$ identity on $\left\{\left(X^{q} \times E^{s}\right)-U_{\varepsilon}\left(K^{p} \times L^{t}, X^{q} \times E^{s}\right)\right\} \times E^{r-s}$, and if

$$
\tilde{x} \in\left(X^{q} \times E^{s}\right) /\left(K^{p} \times L^{t}\right), \quad w \in E^{r-s},
$$

and $\operatorname{pr}_{2}:\left(X^{q} \times E^{s}\right) \times E^{r-s} \rightarrow E^{r-s}$ is the projection onto $E^{r-s}$, then

$$
\left\|w-\operatorname{pr}_{2}(h(\tilde{x}, w))\right\|<\varepsilon .
$$

If, in addition, $Z^{q+s}$ is a compact $(q+s)$-dimensional metric space containing $X^{q} \times E^{s}$, then there exists a homeomorphism $h_{1}: \Sigma^{r-s}\left(Z^{q+s} /\left(K^{p} \times L^{t}\right)\right) \rightarrow \Sigma^{r-s}\left(Z^{q+s}\right)$ such that $h_{1} \circ \Sigma^{r-s} \hat{\rho}=$ identity on $\Sigma^{r-s}\left(Z^{q+s}-U_{\varepsilon}\left(K^{p} \times L^{t}, X^{q} \times E^{s}\right)\right)$, where

$$
\hat{\rho}: Z^{q+s} \rightarrow Z^{q+s} /\left(K^{p} \times L^{t}\right)
$$

is the quotient map.

Furthermore, if $Z^{q+s}$ contains a topological $(q+s)$-cell $\hat{B}^{q+s}$ such that $\left(Z^{q+s}-\right.$ Int $\left.\hat{B}^{q+s}\right) \subset X^{q} \times E^{s}$, where $q+r \geqq 5$ and $q+s \geqq 3$, then there exists a homeomorphism $h_{2}: \Sigma^{r-s}\left(Z^{q+s} /\left(K^{p} \times L^{t}\right)\right) \rightarrow \Sigma^{r-s}\left(S^{q+s}\right) \approx S^{q+r}$ and a flat $(q+s)$-cell $B^{q+s} \subset\left(\right.$ Int $\left.\hat{B}^{q+s}\right)-\left(K^{p} \times L^{t}\right)$ such that $h_{2} \circ \Sigma^{r-s} \hat{\rho}=$ identity on $\Sigma^{r-s} B^{q+s}$, where $B^{q+s}$ is also identified with a flat $(q+s)$-cell in $S^{q+s}$.

Proof. Let $\tilde{K}^{p+t}=K^{p} \times L^{t}$ and $\tilde{X}^{q+s}=X^{q} \times E^{s}$. Since $\tilde{X}^{q+s} \times E^{r-s} \approx E^{q+r}$ and $\widetilde{K}^{p+t}$ can be subdivided so as to be a finite collapsible $(p+t)$-complex, where $(q+s)-(p+t) \geqq 2, q+r \geqq 5$, and $r-s \geqq 1$, the result follows immediately from Theorem 1.6, Corollary 1.8, and [6].

2. The first reduction of the key isotopy theorem. If $X^{q}$ is a separable metric space such that $X^{q} \times E^{r} \approx E^{q+r}$, and $F^{p}$ is a $p$-cell embedded in $X^{q}$, let Hypothesis $(p, q, r)$ denote the statement that $r \geqq 1$ and $F^{p}$ is $(r-1)$-flat in $X^{q} \times E^{r}$. By [6] and [7], we have Hypothesis $(p, q, r)$ if $q-p \neq 1, q+r \geqq 5$, and $r \geqq 1$.

THEOREM 2.1. Suppose $F^{p}$ is a p-cell embedded as a subset of a separable metric space $X^{q}$, where $X^{q} \times E^{r} \approx E^{q+r}$. Let $\alpha$ be the sequence of numbers $a_{1}, b_{1}, a_{2}, b_{2}, \ldots$, $a_{r-1}, b_{r-1}$, where $a_{i}<b_{i}(i=1, \ldots, r-1)$, and let $C_{\alpha}$ denote the $(r-1)$-cell in $E^{r-1}$ given by $C_{\alpha}=\prod_{i=1}^{r-1}\left[a_{i}, b_{i}\right]$ (where $\left[a_{i}, b_{i}\right] \subset i$ th $E^{1}$ factor of $E^{r-1}$ ). If we have 
Hypothesis $(p, q, r)$, then, given real numbers $\varepsilon>0, \delta>0$, and $\eta>0$, there exists an isotopy $f_{t}(t \in[0,1])$ of $\left(X^{q} \times E^{r-1}\right) \times E^{1}$ onto itself such that

(1) $f_{0}=$ identity;

(2) $f_{t} \mid\left(X^{q} \times E^{r}\right)-\left\{U_{\delta}\left(F^{p} \times C_{\alpha}, X^{q} \times E^{r-1}\right) \times E^{1}\right\}=$ identity, for each $t \in[0,1]$;

(3) if $(x, w) \in U_{\delta}\left(F^{p} \times C_{\alpha}, X^{q} \times E^{r-1}\right) \times E^{1}, x \in X^{q}, w \in E^{r}$, then

$$
\left\|w-\operatorname{pr}_{2}\left(f_{t}(x, w)\right)\right\|<\eta
$$

(4) $f_{1}$ is uniformly continuous; and

(5) if $w \in C_{\alpha} \times E^{1} \subset E^{r}$, then $\operatorname{diam} f\left(F^{p} \times w\right)<\varepsilon$.

The proof of this result will follow from a further reduction in §3. In this section, we will show that Theorem $2.1 \Rightarrow$ Theorem 1.1. We note, for $r=1, \alpha$ and $C_{\alpha}$ are not defined, $X^{q} \times E^{0}=X^{q}, F^{p} \times C_{\alpha}=F^{p}$, and $C_{\alpha} \times E^{1}=E^{1}$. Thus, Theorem 2.1, with $r=1$, is equivalent to Theorem 1.1, with $r=1$, and for the remainder of this section we will suppose that $r>1$.

The idea behind the proof that Theorem $2.1 \Rightarrow$ Theorem 1.1 , for $r \geqq 2$, is quite simple. That is, let $R$ denote a rectangular cellular subdivision of $E^{r-1}$ and $R^{i}$ denote the $i$-skeleton of $R$ (i.e., the union of cells of dimension $\leqq i$ ). We will show inductively that Theorem 2.1 allows us to shrink elements of the form $F^{p} \times w$, $w \in\left\{\right.$ a neighborhood of $\left.R^{i-1}\right\} \times E^{1}=N^{i-1} \times E^{1}$, so small, that we can then apply Theorem 2.1 again to shrink elements of the form $F^{p} \times w, w \in$ a neighborhood of $\left.\left(R^{i}-N^{i-1}\right)\right\} \times E^{1}=M^{i} \times E^{1}$, so that the end result is that now all elements of the form $F^{p} \times w, w \in\left\{M^{i} \cup N^{i-1}\right\} \times E^{1} \supset N^{i} \times E^{1}$, are small enough. We now give some details and first obtain some preliminary results.

In what follows, we use the natural Cartesian product metric on $X \times E^{r}$ (or on $X \times E^{r-1}$ ) induced from the given metric on $X$ and the norm metric on $E^{r}$ (or on $\left.E^{r-1}\right)$. We now want to consider a result corresponding to Theorem 2.1 with $C_{\alpha}=\prod_{i=1}^{r-1}\left[a_{i}, b_{i}\right]$ replaced by $N$, where $N$ is an arbitrary subset of $E^{r-1}$. That is, we consider the following statement:

$\left(X^{q}, F^{p}, E^{r}, N\right)_{0}$. Suppose $F^{p}$ is a $p$-cell embedded as a subset of a separable metric space $X^{q}$, where $X^{q} \times E^{r} \approx E^{q+r}$. If we have Hypothesis $(p, q, r)$, then, given $\varepsilon>0, \delta>0, \eta>0$, and $N \subset E^{r-1}$, there exists an isotopy $f_{t}(t \in[0,1])$ of $X^{q} \times E^{r}$ $=\left(X^{q} \times E^{r-1}\right) \times E^{1}$ onto itself such that

(1) $f_{0}=$ identity;

(2) $f_{t} \mid\left(X^{q} \times E^{r}\right)-\left\{U_{\delta}\left(F^{p} \times N, X^{q} \times E^{r-1}\right) \times E^{1}\right\}=$ identity, for each $t \in[0,1]$;

(3) if $(x, w) \in U_{\delta}\left(F^{p} \times N, X^{q} \times E^{r-1}\right) \times E^{1}, x \in X^{q}, w \in E^{r}$, then

$$
\left\|w-\operatorname{pr}_{2}\left(f_{t}(x, w)\right)\right\|<\eta
$$

(4) $f_{1}$ is uniformly continuous; and

(5) if $w \in N \times E^{1} \subset E^{r}$, then $\operatorname{diam} f_{1}\left(F^{p} \times w\right)<\varepsilon$.

We also want to consider two slight modifications of $\left(X^{q}, F^{p}, E^{r}, N\right)_{0}$.

$\left(X^{q}, F^{p}, E^{r}, N\right)_{1}$. Statement $\left(X^{q}, F^{p}, E^{r}, N\right)_{0}$ with " $\ldots$, then there exists . an 
isotopy $f_{t} \ldots$ " replaced by "..., then there exists a real $\zeta>0$ and an isotopy $f_{t} \ldots$ " and (5) replaced by (5) if $w \in N \times E^{1} \subset E^{r}$, then

$$
\operatorname{diam} f_{1}\left(U_{\zeta}\left(F^{p}, X^{q}\right) \times U_{\zeta}\left(w, E^{r}\right)\right)<\varepsilon ;
$$

and

$\left(X^{q}, F^{p}, E^{r}, N\right)_{2}$. Suppose $F^{p}$ is a $p$-cell embedded as a subset of a separable metric space $X^{q}$, where $X^{q} \times E^{r} \approx E^{q+r}$. If we have Hypothesis $(p, q, r)$, then, given $\xi>0$ and $\varepsilon>0$, and $N \subset E^{r-1}$, there exists an isotopy $f_{t}(t \in[0,1])$ of $X \times E^{r}$ $=\left(X \times E^{r-1}\right) \times E^{1}$ onto itself such that

(1) $f_{0}=$ identity;

(2) $f_{t} \mid\left(X^{q} \times E^{r}\right)-\left\{U_{\xi}\left(F^{p}, X^{q}\right) \times U_{\xi}\left(N, E^{r-1}\right) \times E^{1}\right\}=$ identity, for each $t \in[0,1]$;

(3) $)_{2}$ if $w \in E^{r}$, then

$$
f_{t}\left(F^{p} \times w\right) \subset U_{\xi}\left(F^{p}, X^{q}\right) \times U_{\xi}\left(w, E^{r}\right) \text { and }\left\|w-\operatorname{pr}_{2}\left(f_{t}(x, w)\right)\right\|<\xi ;
$$

(4) $f_{1}$ is uniformly continuous; and

(5) if $w \in N \times E^{1}$, then $\operatorname{diam} f_{1}\left(F^{p} \times w\right)<\varepsilon$.

LEMMA 2.2. Suppose that $N$ and $M$ are two arbitrary subsets of $E^{r-1}(r \geqq 2)$. Then (2.2.1) $\left(X^{q}, F^{p}, E^{r}, N\right)_{0} \Rightarrow\left(X^{q}, F^{p}, E^{r}, N\right)_{1}$;

(2.2.2) $\left(X^{q}, F^{p}, E^{r}, N\right)_{0} \Rightarrow\left(X^{q}, F^{p}, E^{r}, N\right)_{2}$; and

(2.2.3) $\left(X^{q}, F^{p}, E^{r}, N\right)_{2}+\left(X^{q}, F^{p}, E^{r}, M\right)_{1} \Rightarrow\left(X^{q}, F^{p}, E^{r}, N \cup M\right)_{0}$.

Proof that $\left(X^{q}, F^{p}, E^{r}, N\right)_{0} \Rightarrow\left(X^{q}, F^{p}, E^{r}, N\right)_{1}$. This is elementary, since $f_{1}$ is uniformly continuous. That is, given $\varepsilon_{1}>0$ in the hypothesis of $\left(X^{q}, F^{p}, E^{r}, N\right)_{1}$, let $\varepsilon_{0}>0$ in the hypothesis of $\left(X^{q}, F^{p}, E^{r}, N\right)_{0}$ be defined by $\varepsilon_{0}=\varepsilon_{1} / 3$. Since $f_{1}$ is uniformly continuous, there exists a $\nu>0$ so that if $Z$ is any subset of $X^{q} \times E^{r}$ having diameter $<\nu$, then $f_{1}(Z)$ has diameter $<\varepsilon_{1} / 3$. Since $\operatorname{diam} f_{1}\left(F^{p} \times w\right)<\varepsilon_{1} / 3$ (for each $\left.w \in N \times E^{1}\right)$, it follows that $\operatorname{diam} f_{1}\left(U_{v}\left(F^{p} \times w, X^{q} \times E^{r}\right)\right.$ ) $<\varepsilon_{1}$ (for each $\left.w \in N \times E^{1}\right)$. Pick $\zeta>0$ so that $U_{\zeta}\left(F^{p}, X^{q}\right) \times U_{\zeta}\left(w, E^{r}\right) \subset U_{v}\left(F^{p} \times w, X^{q} \times E^{r}\right)$ for all $w \in E^{r}$.

Proof that $\left(X^{q}, F^{p}, E^{r}, N\right)_{0} \Rightarrow\left(X^{q}, F^{p}, E^{r}, N\right)_{2}$. Given $\xi>0$, pick $\delta>0$ so that $U_{\delta}\left(F^{p} \times N, X^{q} \times E^{r-1}\right) \subset U_{\xi}\left(F^{p}, X\right) \times U_{\xi}\left(N, E^{r-1}\right)$, and pick $\eta>0$ so that $\eta \leqq \xi$. Let $f_{t}$ be the isotopy of $\left(X^{q}, F^{p}, E^{r}, N\right)_{0}$ satisfying conclusions (1), (2), (3), (4) and (5). The claim is that $f_{t}$ will also satisfy conclusions (1), $(2)_{2},(3)_{2},(4)$ and (5) of $\left(X^{q}, F^{p}, E^{r}, N\right)_{2}$. Clearly, (1), (2) 2 , (4) and (5) are satisfied. We now want to show that $(3)_{2}$ holds.

Since $f_{i}=$ identity outside $U_{\xi}\left(F^{p}, X^{q}\right) \times U_{\xi}\left(N, E^{r-1}\right) \times E^{1}, f_{t}=$ identity outside $U_{\xi}\left(F^{p}, X^{q}\right) \times E^{r}$. Hence, if $\operatorname{pr}_{X^{q}}: X^{q} \times E^{r} \rightarrow X^{q}$ is the projection onto the $X^{q}$ factor, then $\operatorname{pr}_{X^{q}}\left(f_{t}\left(F^{p} \times w\right)\right) \subset U_{\xi}\left(F^{p}, X^{q}\right)$, for all $w \in E^{r}$. Since $\| w-\operatorname{pr}_{2}\left(f_{t}(x, w) \|\right.$ $<\eta \leqq \xi, \quad$ if $\quad(x, w) \in U_{\delta}\left(F^{p} \times N, \quad X^{q} \times E^{r-1}\right) \times E^{1}$, since $\operatorname{pr}_{2}\left(f_{t}(x, w)\right)=w, \quad$ if $(x, w) \notin U_{\delta}\left(F^{p} \times N, X^{q} \times E^{r-1}\right) \times E^{1}$, and $\operatorname{pr}_{2}\left(F^{p} \times w\right)=w$, it follows that

$$
\operatorname{pr}_{2}\left(f_{t}\left(F^{p} \times w\right)\right) \subset U_{\xi}\left(w, E^{r}\right)
$$

for all $w \in E^{r}$. Hence, $f_{t}\left(F^{p} \times w\right) \subset U_{\xi}\left(F^{p}, X^{q}\right) \times U_{\xi}\left(w, E^{r}\right)$ for all $w \in E^{r}$. 
Proof that $\left(X^{q}, F^{p}, E^{r}, N\right)_{2}+\left(X^{q}, F^{p}, E^{r}, M\right)_{1} \Rightarrow\left(X^{q}, F^{p}, E^{r}, N \cup M\right)_{0}$. Let $\varepsilon>0, \delta>0$; and $\eta>0$ be given. We take $(\varepsilon, \delta, \eta)$ in $\left(X^{q}, F^{p}, E^{r}, M\right)_{1}$ to be $(\varepsilon, \delta, \eta / 2)$. Hence, there exists a real number $\xi>0$ and an isotopy $\left(f_{t}\right)_{1}(t \in[0,1])$ of $X^{q} \times E^{r}$ onto itself such that

(1) $\left(f_{0}\right)_{1}=$ identity;

(2) ${ }_{1}\left(f_{t}\right)_{1} \mid\left(X^{q} \times E^{r}\right)-\left\{U_{\delta}\left(F^{p} \times M, X^{q} \times E^{r-1}\right) \times E^{1}\right\}=$ identity $(t \in[0,1])$;

(3) 1 if $(x, w) \in U_{\delta}\left(F^{p} \times M, X^{q} \times E^{r-1}\right) \times E^{1}, x \in X^{q}, w \in E^{r}$, then

$$
\left\|w-\operatorname{pr}_{2}\left(\left(f_{t}\right)_{1}(x, w)\right)\right\|<\eta / 2 ;
$$

(4) ${ }_{1}\left(f_{1}\right)_{1}$ is uniformly continuous; and

$(5)_{1}$ if $w \in M \times E^{1} \subset E^{r}$, then $\operatorname{diam}\left(f_{1}\right)_{1}\left(U_{\xi}\left(F^{p}, X^{q}\right) \times U_{\xi}\left(w, E^{r}\right)\right)<\varepsilon$.

Since $\left(f_{1}\right)_{1}$ is uniformly continuous, there is a $\nu>0$ so that if $Z$ is any subset of $X^{q} \times E^{r}$ having diameter $<\nu$, then $\left(f_{1}\right)_{1}(Z)$ has diameter $<\varepsilon$.

For $(\xi, \varepsilon)$ in $\left(X^{q}, F^{p}, E^{r}, N\right)_{2}$, we take $(\xi, \nu)$, where $\xi>0$ is so small that (i) $U_{\xi}\left(F^{p}, X^{q}\right) \times U_{\xi}\left(N, E^{r-1}\right) \subset U_{\delta}\left(F^{p} \times N, X^{q} \times E^{r-1}\right)$, and (ii) $)_{2} \xi<\min (\eta / 2, \zeta)$. Hence, there exists an isotopy $\left(f_{t}\right)_{2}(t \in[0,1])$ of $X^{q} \times E^{r}$ onto itself such that

$(1)_{2}\left(f_{0}\right)_{2}=$ identity;

(2) ${ }_{2}\left(f_{t}\right)_{2} \mid\left(X^{q} \times E^{r}\right)-\left\{U_{\xi}\left(F^{p}, X^{q}\right) \times U_{\xi}\left(N, E^{r-1}\right) \times E^{1}\right\}=$ identity $(t \in[0,1])$;

(3) $)_{2}$ if $w \in E^{r}$, then

$$
\left(f_{t}\right)_{2}\left(F^{p} \times w\right) \subset U_{\xi}\left(F^{p}, X^{q}\right) \times U_{\xi}\left(w, E^{r}\right) \text { and }\left\|w-\operatorname{pr}_{2}\left(\left(f_{t}\right)_{2}(x, w)\right)\right\|<\xi
$$

for all $t$;

$(4)_{2}\left(f_{1}\right)_{2}$ is uniformly continuous; and

$(5)_{2}$ if $w \in N \times E^{1}$, then $\operatorname{diam}\left(f_{1}\right)_{2}\left(F^{p} \times w\right)<\nu$.

The desired isotopy $\left(f_{t}\right)_{0}$ for $\left(X^{q}, F^{p}, E^{r}, N \cup M\right)_{0}$ is then defined by

$$
\begin{aligned}
\left(f_{t}\right)_{0} & =\left(f_{2 t}\right)_{2}, & & 0 \leqq t \leqq 1 / 2, \\
& =\left(f_{2 t-1}\right)_{1} \circ\left(f_{1}\right)_{2}, & & 1 / 2 \leqq t \leqq 1 .
\end{aligned}
$$

Clearly $\left(f_{0}\right)_{0}=$ identity and $\left(f_{1}\right)_{0}=\left(f_{1}\right)_{1} \circ\left(f_{1}\right)_{2}$ is uniformly continuous. Hence, $(1)_{0}$ and $(4)_{0}$ hold. Since $\left(f_{t}\right)_{2}=$ identity outside $U_{\xi}\left(F^{p}, X^{q}\right) \times U_{\xi}\left(N, E^{r-1}\right)$, and hence $\left(f_{t}\right)_{2}=$ identity outside $U_{\delta}\left(F^{p} \times N, X^{q} \times E^{r-1}\right)$ (by (i) $)_{2}$, and since $\left(f_{t}\right)_{1}$ =identity outside $U_{\delta}\left(F^{p} \times M, X^{q} \times E^{r-1}\right) \times E^{1}$, it follows that $\left(f_{t}\right)_{0}=$ identity outside

$$
\begin{aligned}
\left\{U_{\delta}\left(F^{p} \times N, X^{q} \times E^{r-1}\right) \times E^{1}\right\} \cup\left\{U _ { \delta } \left(F^{p} \times M,\right.\right. & \left.\left.X^{q} \times E^{r-1}\right) \times E^{1}\right\} \\
& =U_{\delta}\left(F^{p} \times(N \cup M), X^{q} \times E^{r-1}\right) \times E^{1} .
\end{aligned}
$$

Hence, (2) 0 holds.

By $(3)_{2}$, since $\xi<\eta / 2$ (by (ii) $\left.)_{2}\right),\left(f_{t}\right)_{2}$ changes $E^{r}$ coordinates $<\eta / 2$. Since by $(3)_{1}$, $\left(f_{t}\right)_{1}$ changes $E^{r}$ coordinates $<\eta / 2$, it follows that $\left(f_{t}\right)_{0}$ changes $E^{r}$ coordinates $<\eta$, and hence $(3)_{0}$ holds.

Finally, we want to show (5) if $w \in(N \cup M) \times E^{1} \subset E^{r}$, then $\operatorname{diam}\left(f_{1}\right)_{0}\left(F^{p} \times w\right)$ $<\varepsilon$. If $w \in N \times E^{1}$, then $\operatorname{diam}\left(f_{1}\right)_{2}\left(F^{p} \times w\right)<\nu$ (by $\left.(5)_{2}\right)$; hence

$$
\operatorname{diam}\left(f_{1}\right)_{1} \circ\left(f_{1}\right)_{2}\left(F^{p} \times w\right)<\varepsilon,
$$


by our choice of $\nu$. Now suppose $w \in M \times E^{1}$. Then

$$
\left(f_{1}\right)_{2}\left(F^{p} \times w\right) \subset U_{\xi}\left(F^{p}, X^{q}\right) \times U_{\xi}\left(w, E^{r}\right) \subset U_{\zeta}\left(F^{p}, X^{q}\right) \times U_{\zeta}\left(w, E^{r}\right),
$$

by the first part of (3) $)_{2}$ and our choice of $\xi$ (by (ii) $)_{2}$ ). Since by (5) $)_{1}$, if $w \in M \times E^{1}$, then $\operatorname{diam}\left(f_{1}\right)_{1}\left(U_{\zeta}\left(F^{p}, X^{q}\right) \times U_{\zeta}\left(w, E^{r}\right)\right)<\varepsilon$, it follows that $\left(f_{1}\right)_{1} \circ\left(f_{1}\right)_{2}\left(F^{p} \times w\right)<\varepsilon$ (for $w \in M \times E^{1}$ ). Hence, (5), holds and our proof that

$$
\left(X^{q}, F^{p}, E^{r}, N\right)_{2}+\left(X^{q}, F^{p}, E^{r}, M\right)_{1} \Rightarrow\left(X^{q}, F^{p}, E^{r}, N \cup M\right)_{0}
$$

is now complete.

Proof that Theorem 2.1 $\Rightarrow$ Theorem 1.1. We will show that if Theorem 2.1 is true, then statement $\left(X^{q}, F^{p}, E^{r}, N\right)_{0}$ is valid for $N=E^{r-1} \subset E^{r-1} \times E^{1}=E^{r}$. Clearly, this implies that Theorem 1.1 is true.

We first consider $I^{r-1}=[0,1]^{r-1} \subset E^{r-1}$. Let $I^{r-1}$ have the natural cellular subdivision consisting of the various $m$-faces of $I^{r-1}(m=0,1, \ldots, r-1)$. Let $R_{\boldsymbol{\theta}_{r-1}}$ denote the collection of all $m$-cells $C^{m} \subset I^{r-1}(0 \leqq m \leqq r-1)$ containing the origin $\Theta_{r-1}$ of $E^{r-1}$. Let $R^{0}=\left\{\left(a_{1}, \ldots, a_{r-1}\right) \in E^{r-1} \mid\right.$ each $a_{j}$ is an integer $\}$. Our cellular subdivision $R$ of $E^{r-1}$ is defined as follows:

$$
R=\left\{C_{a}^{m} \mid C_{a}^{m}=C^{m}+a, 0 \leqq m \leqq r-1, a \in R^{0}, \text { and } C^{m} \in R_{\boldsymbol{\Theta}_{r-1}}\right\} .
$$

Let $\left(a_{1}, \ldots, a_{r-1}\right)=a \in R^{0}$ denote an arbitrary 0-cell of $R$. Let

$$
N_{a}^{0}=\prod_{j=1}^{r-1}\left[a_{j}-1 / 4, a_{j}+1 / 4\right]
$$

and let

$$
N^{0}=\bigcup\left\{N_{a}^{0} \mid a \in R^{0}\right\} .
$$

If $0<\delta \leqq 1 / 8$, we can apply Theorem 2.1 locally (and in a uniform manner) to each $U_{\delta}\left(F^{p} \times N_{a}^{0}, X^{q} \times E^{r-1}\right) \times E^{1}$ and obtain $\left(X^{q}, F^{p}, E^{r}, N^{0}\right)_{0}$. We can guarantee that the isotopy is uniform by obtaining an appropriate isotopy on

$$
U_{\delta}\left(F^{p} \times N_{\Theta_{r-1}}^{0}, X^{q} \times E^{r-1}\right)+E^{1},
$$

and then translating this single isotopy to the other $U_{\delta}\left(F^{p} \times N_{a}^{0}, X^{q} \times E^{r-1}\right) \times E^{1}$, for each $a \in R^{0}$, we obtain our first claim.

Let $R^{1}$ denote the 1 -skeleton of our cellular subdivision $R$ of $E^{r-1}$. Then $R^{1}$ - Int $N^{0}$ is a countable collection of disjoint segments, and they lie in a countable collection of disjoint rectangular $(r-1)$-cells having faces parallel to the various $(r-2)$-hyperplanes determined by the equations $x_{j}=0(j=1,2, \ldots, r-1)$. We denote the union of these $(r-1)$-cells by $M^{1}$. Again applying Theorem 2.1 locally, it follows that $\left(X^{q}, F^{p}, E^{r}, M^{1}\right)_{0}$ holds (it suffices to only consider those $(r-1)$-cells of $M^{1}$ which are compact neighborhoods of the corresponding segments lying in the "1-skeleton" of $R_{\boldsymbol{\theta}_{r-1}}$, and then translate this finite collection of isotopies by the points $\left.a \in R^{0}\right)$. By Lemma 2.2, (2.2.1)-(2.2.3), we see that $\left(X^{q}, F^{p}, E^{r}, M^{1}\right)_{1},\left(X^{q}, F^{p}, E^{r}, N^{0}\right)_{2}$, and $\left(X^{q}, F^{p}, E^{r}, N^{0} \cup M^{1}\right)_{0}$ hold. 
Let $\tilde{N}^{1}$ be a "nice" rectangular neighborhood of $R^{1}$ so that $\tilde{N}^{1} \subset M^{1} \cup N^{0}=N^{1}$ and $R^{2}$-Int $\tilde{N}^{1}$ is a countable collection of disjoint rectangular disks. We then can obtain an $M^{2}$ consisting of the union of a countable collection of disjoint rectangular $(r-1)$-cells containing the rectangular disks above. As above, we can obtain $\left(X^{q}, F^{p}, E^{r}, N^{1} \cup M^{2}\right)_{0}$.

Repeating this process, we can finally obtain $\left(X^{q}, F^{p}, E^{r}, N^{r-2} \cup M^{r-1}\right)_{0}$ $=\left(X^{q}, F^{p}, E^{r}, E^{r-1}\right)$. Hence, the proof that Theorem $2.1 \Rightarrow$ Theorem 1.1 follows by induction, using the $i$-skeleton $R^{i}$ of $R(0 \leqq i \leqq r-1)$.

3. The second reduction of the key isotopy theorem. Given integers $p$, and $r$ $(1 \leqq p \leqq q$ and $r \geqq 1)$, let $I^{p+r-1}=\left\{\left(x_{1}, \ldots, x_{q+r}\right) \in E^{q+r} \mid 0 \leqq x_{i} \leqq 1\right.$ for $1 \leqq i \leqq p+r-1$, and $x_{i}=0$ for $\left.p+r-1<i \leqq q+r\right\}$. If $m$ is an integer, such that $1 \leqq m \leqq p$, and we set $k=p+r-1$, let $I^{k}=I^{m} \times I^{k-m}$, where $I^{m}=\left\{\left(x_{1}, \ldots, x_{q+r}\right) \in I^{k} \mid x_{i}=0\right.$ for $m<i \leqq q+r\}$ and $I^{k-m}=\left\{\left(x_{1}, \ldots, x_{q+r}\right) \in I^{k} \mid x_{i}=0\right.$ for $1 \leqq i \leqq m$ and for $\left.k<i \leqq q+r\right\}$ (if $r=1$ and $m=p, I^{k}=I^{p} \times I^{0}=I^{p}$ ).

Let $\alpha$ be a fixed sequence of real numbers $a_{1}, b_{1}, a_{2}, b_{2}, \ldots, a_{r-1}, b_{r-1}$, where $a_{i}<b_{i}(i=1, \ldots, r-1)$, and let $C_{\alpha}$ denote the $(r-1)$-cell in $E^{r-1} \times 0 \subset E^{r}$ given by $C_{\alpha}=\left(\prod_{i=1}^{r-1}\left[a_{i}, b_{i}\right]\right) \times 0$, where $\left[a_{i}, b_{i}\right]$ is a subinterval of the $i$ th $E^{1}$ factor of $E^{r-1} \times 0$. (If $r=1, \alpha$ and $C_{\alpha}$ are not defined and $E^{r}=E^{r-1} \times E^{1}=E^{0} \times E^{1}=E^{1}$; also $X^{q} \times E^{r-1}=X^{q} \times E^{0}=X^{q}$.) If $I_{i}^{1}$ denotes the $i$ th $I^{1}$ factor of $I^{k-p}$ (the $(p+i)$ th $I^{1}$ factor of $I^{k}$ ), let $g_{\alpha, i}: I_{i}^{1} \rightarrow\left[a_{i}, b_{i}\right] \subset C_{\alpha}$ be the homeomorphism defined by $g_{\alpha, i}\left(x_{p+i}\right)=\left(1-x_{p+i}\right) a_{i}+\left(x_{p+i}\right) b_{i}(1 \leqq i \leqq r-1)$ and let $g_{\alpha} ; I^{k-p} \rightarrow C_{\alpha}$ be the homeomorphism defined by $g_{\alpha}=\left(\prod_{i=1}^{r-1} g_{\alpha, i}\right):\left(\prod_{i=1}^{r-1} I_{i}^{1}\right) \rightarrow C_{\alpha}$.

THEOREM 3.1. Suppose $F^{p}$ is a p-cell embedded as a subset of a separable metric space $X^{q}$, where $X^{q} \times E^{r} \approx E^{q+r}$. Let $k=p+r-1$ and let $I^{k}=I^{p} \times I^{k-p}, C_{\alpha}$, and $g_{\alpha}: I^{k-p} \rightarrow C_{\alpha}$ be as above. Let $g_{p}: I^{p} \rightarrow F^{p}$ be a homeomorphism carrying $I^{p}$ onto $F^{p}$ and let $g=\left(g_{p} \times g_{\alpha}\right): I^{p} \times I^{k-p} \rightarrow F^{p} \times C_{\alpha}$ denote the induced homeomorphism carrying $I^{k}$ onto $F^{p} \times C_{\alpha}$. If we have Hypothesis $(p, q, r)$ (refer to §2), then, given $\varepsilon>0, \delta>0, \eta>0$, and an integer $m$ such that $1 \leqq m \leqq p$, there exists an isotopy $h_{t}$ $(t \in[0,1])$ of $X^{q} \times E^{r}$ onto itself such that

(1) $h_{0}=$ identity;

(2) $h_{t}=$ identity outside $U_{\delta}\left(g\left(I^{k}\right), X^{q} \times E^{r-1}\right) \times E^{1}$, for each $t \in[0,1]$;

(3) $h_{t}$ changes $E^{r}$ coordinates $<\eta$, for each $t \in[0,1]$;

(4) $h_{1}$ is uniformly continuous;

(5) diam $h_{1}\left(g\left(I^{m} \times x\right) \times w\right)<\varepsilon$, for each $x \in I^{k-m}$ and each $w \in E^{1}$; and

(6) for each $w \in E^{1}$, there exists $y \in I^{m}$ such that

$$
h_{1}\left(g\left(I^{m} \times x\right) \times w\right) \subset U_{\varepsilon}\left(g(y, x) \times w, X^{q} \times E^{r}\right),
$$

for each $x \in I^{k-m}$.

Clearly, the first five conclusions of Theorem 3.1 , when $m=p$, immediately give us Theorem 2.1. Theorem 3.1 is essentially statement $H(n, k, m)$ proved in [3]. By using induction on $m$, it is not too difficult to show that Theorem 3.1, with 
$1 \leqq m \leqq p$, follows directly from the corresponding result with $m=1$ only. In fact, the appropriate argument is given explicitly in the proof of Lemma 2.1 of [3]. We conclude our work here by observing that Theorem 3.1, with $m=1$, follows directly from the remainder of the work done in [3], plus parts of [1] and [2].

That is, implicit in the proof of the main result of [3] is the following:

$\left.{ }^{*}\right)$ If $g: I^{p} \times I^{k-p} \rightarrow E^{n}$ is an embedding such that $g\left(I^{p} \times I^{k-p}\right)$ is flat in $E^{n+1}=E^{n} \times E^{1}$, then the usc decomposition $G$ of $E^{n} \times E^{1}$, whose nondegenerate elements are of the form $g\left(I^{p} \times x\right) \times w\left(x \in I^{k-p}, w \in E^{1}\right)$ is shrinkable. Moreover, the shrinking isotopies can be chosen so that the distance from a point $y \in E^{n+1}$ to any $g\left(I^{p} \times x\right) \times w$ will not be changed appreciably.

We simply need to observe here that if $X^{n} \times E^{1} \approx E^{n+1}$, then the above statement holds for embeddings $g: I^{p} \times I^{k-p} \rightarrow X^{n}$ such that $g\left(I^{p} \times I^{k-p}\right)$ is flat in $E^{n+1}$. This follows since the arguments used in [3] apply as given as long as we have this flatness condition and the given product structure $X^{n} \times E^{1}$ on $E^{n+1}$. Finally, we see that Theorem 3.1 holds by setting $X^{n}=X^{q} \times E^{r-1}$ and

$$
g=g_{p} \times g_{\alpha, 1} \times \cdots \times g_{\alpha, r-1}: I^{p} \times I \times \cdots \times I \rightarrow F^{p} \times\left[a_{1}, b_{1}\right] \times \cdots \times\left[a_{r-1}, b_{r-1}\right] .
$$

Because of the canonical manner in which the $I^{k-p}$ factor of $I^{k}$ is embedded in $E^{r-1}$, the "moreover" part of $\left(^{*}\right)$ will guarantee that conclusion (3) of Theorem 3.1 will hold. This completes our discussion of Theorem 3.1. For further details, the reader should refer to [3].

\section{REFERENCES}

1. J. J. Andrews and M. L. Curtis, n-space modulo an arc, Ann. of Math. (2) 75 (1962), 1-7. MR 25 \#2590.

2. R. H. Bing, The cartesian product of a certain nonmanifold and a line is $E^{4}$, Ann. of Math. (2) 70 (1959), 399-412. MR 21 \#5953.

3. John L. Bryant, Euclidean space modulo a cell, Fund. Math. 63 (1968), 42-51. MR 37 \#5861.

4. - Euclidean $n$-space modulo an ( $n-1)$-cell (to appear).

5. J. L. Bryant and C. L. Seebeck III, Locally nice embeddings of polyhedra, Quart. J. Math. Oxford Ser. (2) 19 (1968), 257-274. MR 38 \#2751.

6. _ Locally nice embeddings in codimension three, Bull. Amer. Math. Soc. 74 (1968), 378-380. MR 36 \#4566.

7. A. V. Černavskiĭ, Locally homotopically unknotted embeddings of manifolds, Dokl. Akad. Nauk SSSR 181 (1968), 290-293 =Soviet Math. Dokl. 9 (1968), 835-839. MR 38 \#720.

8. —_ Topological imbeddings of manifolds, Dokl. Akad. Nauk SSSR 187 (1969), 1247$1250=$ Soviet Math. Dokl. 10 (1969), 1037-1041.

9. L. C. Glaser, An elementary decomposition proof that the double suspension of a homotopy 3-cell is a topological 5-cell, Illinios J. Math. (to appear).

10. - On suspensions of homology spheres, Proc. 1970 NUFFIC International Summer School on Manifolds (to appear).

11. D. S. Gillman, Unknotting 2-manifolds in 3-hyperplanes in $E^{4}$, Duke Math. J. 33 (1966), 229-245. MR 32 \#6441.

12. T. Homma, Piecewise linear approximations of embeddings of manifolds, Florida State University, Tallahassee, Fla., 1965 (mimeographed notes). 
13. W. Hurewicz and H. Wallman, Dimension theory, Princeton Math. Series, vol. 4, Princeton Univ. Press, Princeton, N. J., 1941. MR 3, 312.

14. V. L. Klee, Some topological properties of convex sets, Trans. Amer. Math. Soc. 78 (1955), 30-45. MR 16, 1030.

15. C. Lacher, Locally flat strings and half-strings, Proc. Amer. Math. Soc. 18 (1967), 299304. MR 35 \#3670.

16. Richard T. Miller, Approximating codimension-3 embeddings, Notices Amer. Math. Soc. 17 (1970), 470. Abstract \#70 T-G47.

INSTITUTE fOR AdVANCEd StUdy,

Princeton, New Jersey 08540

UNIVERSITY OF UTAH,

Salt Lake City, Utah 84112 